\title{
L'Effet de l'Âge sur la Distribution des Salaires
}

\author{
Sarah Le Duigou \\ GAINS-TEPP \\ Université du Maine
}

The distribution of wages varies with workers'age. In this article we present the principles which allow to build a model able to explain this evolution. This model is based on the search model developed by Burdett and Mortensen (1998). It considers two age groups, the juniors and the seniors and takes into account three channels of wage evolution: the job-to-job mobility, the evolution of the balance of power, and the human capital accumulation. Using US data, we show that these three channels allow to reproduce quite well the aggregated wage distribution as well as the evolution of it over the workers life-cycle. The channels of job-to-job mobility and the evolution of the balance of power account for the lower density of low wages in the seniors' wage distribution and the human capital accumulation for the lower density of very high wages in the juniors' wage distribution.

La distribution des salaires varie avec l'âge des travailleurs. Dans cet article, nous présentons les principes permettant de construire un modèle en mesure d'expliquer cette évolution. Fondé sur le cadre théorique de Burdett et Mortensen (1998), ce modèle considère deux périodes de vie, celle des juniors et celle des seniors. Il prends en compte trois canaux d'évolution des salaires : la mobilité professionnelle ascendante, l'évolution du rapport de force, et l'accumulation de capital humain. Sur données Américaines, nous montrons que ces trois canaux permettent de reproduire en même temps la distribution agrégée des salaires ainsi que son évolution au cours du cycle de vie des travailleurs. Les deux premiers canaux permettent d'expliquer la baisse du nombre des bas salaires avec l'âge. L'accumulation de capital humain, elle, explique pourquoi les seniors détiennent la majorité des très hauts salaires. 
La distribution des salaires, tout comme d'autres statistiques du marché du travail, varie avec l'âge. Aux États-Unis, le salaire moyen des seniors est $12 \%$ plus élevé de celui des jeunes et la dispersion salariale passe d'un écart-type de 1,6 à 1,8 (cf. Figure 1). L'objectif de cet article est d'exposer les principes qui ont permis de construire un modèle structurel permettant d'expliquer cette évolution et susceptible de servir de base à l'évaluation de politiques publiques pouvant avoir des effets différenciés par âge ${ }^{1}$.

\section{Figure 1}

Distribution des salaires des hommes aux États-Unis en fonction de leur âge

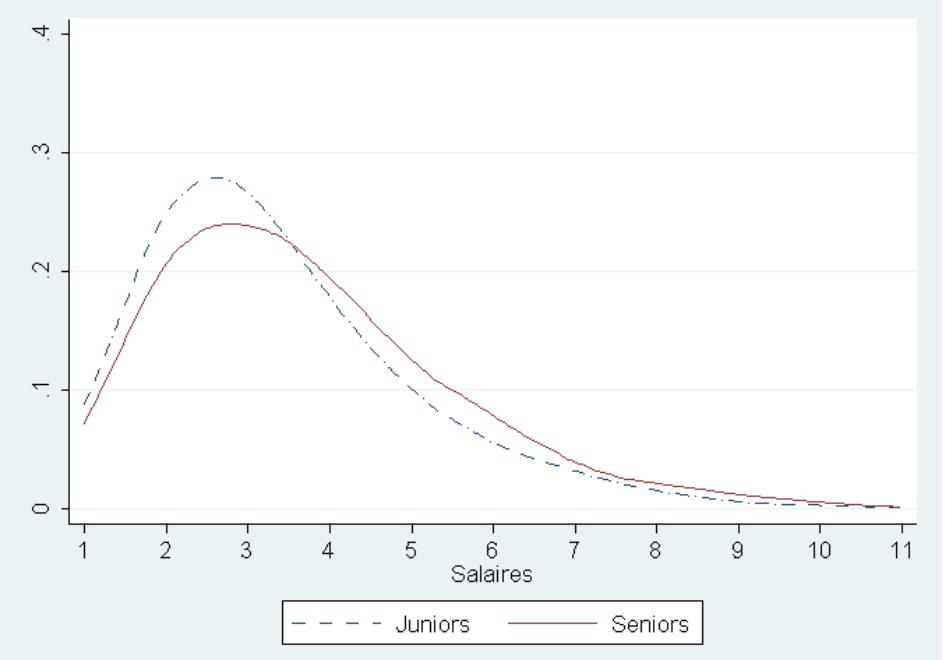

Lecture : les juniors sont constitués des 20-43 ans et les seniors des 43-66 ans . Les salaires sont des salaires horaires et sont exprimés relativement au niveau du salaire horaire minimum fédéral. Champ : hommes salariés, tout contrat, âgés de 20 à 66 ans.

Source : Current Population Survey de 2002.

Il existe trois canaux principaux permettant d'expliquer cette évolution des salaires avec l'âge. D'abord, grâce aux mobilités d'emploi à emploi, le temps passé sur le marché du travail donne aux travailleurs plus de chances de recevoir des bonnes opportunités d'emploi. Les travailleurs quittent progressivement les emplois les moins bien payés pour aller vers les mieux payés. C'est le canal de la mobilité professionnelle ascendante. Ensuite, au cours du cycle de vie, à mesure que le salaire de réserve des travailleurs en emploi augmente, les travailleurs gagnent en pouvoir de marché sur les entreprises qui sont contraintes d'offrir des salaires supérieurs pour les attirer. C'est le canal de l'évolution du rapport de force. Enfin, les travailleurs apprennent au cours de leur vie active, leur productivité tend donc à s'accroître. C'est le canal de l'accumulation de capital humain général. Cet article se propose d'évaluer la contribution de chacun de ces trois canaux à la distribution des salaires et à son évolution.

Depuis l'article de Burdett et Mortensen (1998), il est d'usage d'utiliser le modèle de recherche d'emploi avec friction pour expliquer la distribution des salaires. Dans ce cadre théorique, les travailleurs cherchent un emploi qu'ils soient chômeurs ou déjà en emploi. Les firmes ${ }^{2}$ sont en concurrence entre elles pour recruter et retenir les travailleurs. Leurs propositions de salaires prennent en compte la répartition des salaires de réservation des travailleurs et les salaires offerts par les autres entreprises. Les frictions sur le marché du travail donnent un certain pouvoir de monopsone aux firmes via deux effets : d'abord les travailleurs ne trouvent pas instantanément un

${ }^{1}$ Cet article s'appuie sur un modèle structurel qui est développé dans le document de travail « The Effect of Age on the Wage Distribution : A Quantitative Evaluation Using US Data » en ligne sur le site de TEPP. Le modèle développé dans ce document est d'ordre plus général et contient quelques extensions qui n'ont pas pu être exposées dans ce présent article. Le principe du modèle reste cependant le même.

2 Dans ce modèle, on considère qu'une firme n'est constituée que d'un poste 
travail quand ils sont au chômage, cela génère une armée de réserve de chômeurs prêts à accepter le salaire minimum, ensuite les travailleurs ne trouvent pas instantanément un meilleur poste quand ils sont en emploi, cela amoindrit la concurrence entre les firmes pour retenir leur employé. En effet, un risque de débauchage plus faible permet aux firmes d'être moins confrontées à la concurrence des autres firmes et donc de pouvoir pratiquer des salaires plus bas. Les frictions rationalisent donc l'existence de propositions de salaire au niveau du salaire minimum. Le résultat de ce jeu non coopératif d'offre de salaire mène à un équilibre en stratégie mixte dans lequel les firmes répartissent leurs offres sur un intervalle de salaires. Pour une même espérance de profit, plusieurs stratégies d'offre de salaires sont possibles : un salaire en bas de distribution permet de dégager un profit instantané élevé mais suppose une procédure de recrutement longue et une rétention faible du travailleur, alors qu'un salaire en haut de distribution génère un profit instantané limité mais une bonne rétention du travailleur et un recrutement rapide. Mortensen (1998) étend ce cadre théorique en introduisant une hétérogénéité endogène des firmes, qui peuvent choisir d'augmenter leur productivité sur un appariement donné, en s'acquittant d'un investissement initial au début de l'appariement. A l'équilibre, les firmes emploient des travailleurs ayant des niveaux de capital humain spécifique $e^{3}$ différents et ont en conséquence des productivités différentes. Le modèle de Burdett et Mortensen (1998) augmenté de l'hétérogénéité des appariements proposée par Mortensen (1998) permet de reproduire de façon crédible la distribution observée des salaires.

Toutefois, ce modèle en horizon infini ne permet pas d'expliquer comment le jeu des salaires se modifie avec l'âge des travailleurs. Dans cet article ${ }^{4}$, nous penons donc en compte le cycle de vie des travailleurs en considérant deux périodes de vie: la période «junior » et la période "senior ». Les travailleurs ont ainsi désormais un horizon de vie fini puisqu'à l'issue de cette deuxième période, les travailleurs se retirent du marché du travail.

Les travailleurs commencent leur vie active comme juniors et au chômage. Grâce aux mobilités chômage-emploi, ce chômage d'insertion sur le marché du travail se résorbe peu à peu entre les deux périodes. Dans le modèle de Burdett et Mortensen (1998), la modélisation de la recherche en emploi permet déjà de garantir une trajectoire croissante des salaires avec le temps passé sur le marché du travail, puisque chaque travailleur ne quitte son emploi qu'à la suite d'une offre plus avantageuse. Par le jeu des mobilités professionnelles ascendantes, les travailleurs dans la période « senior » auront des salaires en moyenne plus élevés que ceux dans leur période « junior ».

Les firmes dirigent leurs recherches sur l'un ou l'autre des segments de travailleurs. Une offre d'emploi stipule en effet souvent si l'entreprise désire quelqu'un de peu expérimenté (junior) ou d'expérimenté (senior). Les deux types de travailleurs ne sont pas substituables pour la firme. Un junior possède des caractéristiques, par exemple de résistance physique, qu'un senior n'aura pas, et un senior possède des caractéristiques, par exemple intellectuelles ou d'expérience, qu'un junior n'aura pas. Étant donné la hausse de salaire induite par les mobilités entre les deux périodes et la baisse du chômage, les firmes auront sur le segment "senior» un moindre pouvoir de monopsone et la concurrence qu'elles se livreront sur ce segment les amènera à augmenter d'avantage leurs salaires. Cette hypothèse de recherche dirigée de la part des firmes permet de rendre compte de l'effet de l'évolution du rapport de force entre firmes et travailleurs sur la distribution des salaires.

Le pouvoir de marché des travailleurs en période "senior» tend toutefois à être amoindri par leur horizon plus court. Cet effet est modélisé par l'extension de Mortensen (1998). Si les firmes peuvent investir en capital humain spécifique dans leur appariement afin de le rendre plus productif, elles seront nécessairement moins enclines à le faire avec un travailleur approchant de la retraite et qui a donc un horizon d'amortissement de cet investissement plus court. Les firmes se feront donc une

3 Ce capital humain spécifique n'est pas transférable d'un emploi à l'autre, il ne doit donc pas être confondu avec le capital humain général aussi présent dans ce modèle.

4 En étendant le modèle de Burdett et Mortensen (1998), nous faisons l'hypothèse que les firmes ne peuvent proposer qu'un contrat à un salaire. 
concurrence moins vive pour attirer ces travailleurs qui leur apportent une moindre productivité.

A ce stade de la modélisation, il est possible de tester la capacité du modèle de recherche d'emploi avec friction en horizon fini à expliquer l'évolution du jeu de salaire avec l'âge. Un des résultats de cet article est que le jeu de concurrence entre les firmes ne permet pas de reproduire à lui seul le déplacement vers la droite de la distribution des salaires ni l'étalement de la distribution (la hausse de l'écart-type). Ce modèle sous-estime donc la contribution de la hausse des salaires au cours du cycle de vie à la dispersion totale des salaires.

Nous introduisons donc le canal de l'accumulation de capital humain général au cours du cycle de vie qui est indispensable pour rendre compte de cette hausse et expliquer la totalité de l'évolution de la distribution des salaires avec l'âge. Ce canal est introduit en considérant que les travailleurs accumulent entre les deux périodes de façon exogène du capital humain général. Les travailleurs sont donc hétérogènes pour les firmes par trois aspects : leur âge, leur capital humain spécifique qui a été investi par la firme au début de l'appariement et leur capital humain général.

Les trois canaux, mobilité professionnelle ascendante, évolution du rapport de force et accumulation $d u$ capital humain général permettent de reproduire la distribution des salaires agrégée et les différences majeures en termes de moyennes, médianes et variances des salaires entre les deux périodes. Étant donné que ce modèle ne prend pas en compte d'hétérogénéité ex ante des travailleurs (formation initiale), la reproduction de la distribution des salaires de travailleurs plutôt homogènes est meilleure que celle de la distribution des salaires de la population totale.

Ces trois canaux expliquent des aspects différents de ces distributions. Selon les résultats des simulations effectuées dans cet article, les mobilités ascendantes et la hausse du rapport de force permettent d'expliquer pourquoi les travailleurs seniors sont relativement moins affectés par de bas salaires. L'intuition est que ces salaires ne leur sont en fait pas proposés puisqu'ils visent essentiellement les chômeurs ou les travailleurs qui n'ont connu aucune augmentation de salaire et qui ont donc un faible pouvoir de marché. Toutefois ces deux canaux ne parviennent pas à expliquer que les travailleurs seniors bénéficient de la majorité des salaires les plus élevés. Cette réalité est expliquée par l'accumulation du capital humain général et donc la meilleure productivité de ces travailleurs expérimentés.

L'étude de l'effet de l'âge sur les distributions de salaires fait l'objet d'une littérature très récente mais assez restreinte. Le manuscrit de Bagger et al. (2007) explique la progression salariale via les mobilités ascendantes et l'accumulation de capital humain dans le cadre théorique développé par Postel-Vinay et Robin (2002) mais en horizon infini. Menzio et al. (2012) traite de ce lien en horizon fini en distinguant ces deux mêmes canaux d'évolution des salaires. Il rend compte de leurs effets sur les transitions emploi-chômage, chômage-emploi et emploi-emploi ainsi que sur le profil des salaires moyens au cours du cycle de vie. Les auteurs se placent dans un cadre théorique très différent de celui de Burdett et Mortensen (1998) : dans leur modèle, tous les agents se comportent de façon efficiente et l'information est parfaite. Le marché du travail est divisé en sous-marchés du travail dans lesquels se rencontrent des travailleurs qui ne recherchent qu'un seul salaire et ou seulement un salaire est proposé. La recherche est donc parfaitement dirigée des deux côtés, celui des travailleurs et des firmes. En conséquence, les firmes n'ont pas de pouvoir de monopsone et l'effet des frictions est beaucoup moins fort que dans notre cadre théorique. L'article de Menzio et al. (2012) contribue également à une littérature dans laquelle les contrats peuvent être constitués d'une trajectoire de salaires s'étalant dans le temps (cf. Burdett et Coles, (2003)). L'article que nous présentons ici, ne s'inscrit pas dans cette littérature.

La première section présentera les hypothèses et les mécanismes importants du modèle théorique. La seconde section présentera la calibration de ce modèle sur données américaines. La dernière 
section conclura.

\section{Section 1 : Modèle théorique}

\subsection{Hypothèses du modèle}

Le cycle de vie des travailleurs est découpé en deux périodes de même durée: les juniors sont les travailleurs entrant sur le marché du travail, ils deviennent ensuite des seniors avec une certaine probabilité. Cette probabilité assure qu'en espérance les travailleurs restent juniors la première moitié de leur vie active et seniors la deuxième moitié. A l'issue de ces deux périodes, ils se retirent de la vie active. Entre la première et la deuxième période, les travailleurs acquièrent un capital humain général qui est exogène.

En dehors de l'aspect cycle de vie et de la présence de capital humain général, le modèle reprend les hypothèses clés du modèle de Burdett et Mortensen (1998) augmenté de l'hétérogénéité endogène des appariements de Mortensen (1998).

La seule action des travailleurs est la recherche d'emploi qu'ils peuvent effectuer aussi bien au chômage qu'en emploi. Le salaire de réservation d'un chômeur est le coût d'opportunité du travail et celui d'un employé son salaire courant. A l'entrée du marché du travail, les travailleurs sont tous homogènes et au chômage, mais du fait des aléas de mobilité, chômage-emploi, emploi-emploi, emploi-chômage, ils ont à l'équilibre un salaire de réserve différent.

Les firmes sont en concurrence entre elles pour recruter et retenir les travailleurs sur chacun des segments de travailleurs, les juniors et les seniors. Elles peuvent donc diriger leur recherche selon le critère de l'âge, mais ne peuvent par contre pas la diriger selon le salaire de réservation des travailleurs, ni selon leur statut, l'information étant imparfaite. Les firmes peuvent créer à l'embauche des emplois plus ou moins productifs en fonction d'un investissement initial qui peut être assimilé à de la formation spécifique du travailleur sur le poste. La production générée par l'appariement dépend donc de cet investissement initial et reste constante tout au long de l'appariement.

Il existe du chômage dans cette économie du fait de l'existence de frictions : les travailleurs ne trouvent pas instantanément du travail lorsqu'ils deviennent chômeurs. Les travailleurs entrant sur le marché du travail mettent donc un certain temps avant de trouver leur premier emploi. De plus, avec une certaine probabilité, les emplois sont détruits par des chocs exogènes. Le taux d'arrivée de ces chocs est constant au cours de la vie des travailleurs.

\subsection{Le cycle de vie et la mobilité des travailleurs}

Faisons pour le moment l'hypothèse que les travailleurs font face, quel que soit leur âge, juniors ou seniors, aux mêmes propositions de salaires de la part des entreprises afin de comprendre le seul effet des mobilités au cours du cycle de vie. Nous relâcherons cette hypothèse plus loin pour étudier le rôle de l'évolution du rapport de force et de l'accumulation du capital humain général.

Les travailleurs juniors en emploi n'ont pour la plupart pas encore eu le temps de s'être vu proposer beaucoup de bonnes opportunités salariales pendant leur relativement courte expérience sur le marché du travail. Les travailleurs seniors ont eux eu toute la première partie de leur vie (ils étaient alors juniors) pour monter dans l'échelle des salaires grâce à un plus grand nombre de mobilités d'emploi à emploi et sont donc plus nombreux à toucher de hauts salaires. Notons qu'individuellement un junior peut avoir de la chance dès son premier emploi et trouver un salaire supérieur à beaucoup de seniors, toutefois en espérance les salaires des seniors seront supérieurs.

Grâce aux mobilités chômage-emploi et emploi-emploi, le temps passé sur le marché du travail améliore globalement la situation des travailleurs : au cours de la vie, les travailleurs sortent du 
chômage d'insertion sur le marché du travail et sont employés par les firmes offrant de plus hauts salaires.

\subsection{Comportement des firmes et équilibre}

Les firmes maximisent leur profit en se faisant une concurrence à la Bertrand. En plus du salaire, elles peuvent utiliser un levier supplémentaire dans ce jeu concurrentiel, l'investissement initial dans l'appariement qui permet d'augmenter la productivité. Elles cherchent donc un niveau de salaire et un niveau d'investissement initial qui maximisent leur profit espéré étant donnés la distribution des salaires proposés par les autres firmes et celle des salaires de réservation des travailleurs. Burdett et Mortensen (1998) montrent que le résultat de ce jeu de salaires est une distribution de salaires. L'intuition de ce résultat est la suivante: si toutes les entreprises fixent leur salaire au niveau du salaire de réservation des chômeurs, une entreprise aura forcément intérêt à dévier en offrant un salaire très légèrement supérieur et pouvoir par là même débaucher tous les travailleurs en emploi. Les firmes vont donc se répartir pour chaque âge sur le support allant du salaire de réservation des chômeurs au salaire maximal défini par le modèle comme garantissant l'équiprofit. Les firmes vont donc adopter des stratégies salariales différentes mais à l'équilibre stationnaire, au sein de chaque classe d'âge, elles auront toutes la même espérance de profit.

Dans cette sous-section, nous nous intéressons aux stratégies salariales les plus profitables pour les firmes de chaque classe d'âge. En effet les salaires les plus profitables pour les firmes seront ceux offerts par un plus grand nombre de firmes ${ }^{5}$ ce qui affectera directement la forme des distributions de salaires. Le profit espéré dépend de quatre éléments: la probabilité de recrutement d'un travailleur, le profit que ce travailleur générera à chaque instant, la durée de l'emploi et le montant de l'investissement initial en formation spécifique. La façon dont le salaire agit sur ces quatre éléments détermine le comportement salarial des firmes et donc l'état du rapport de force entre les firmes et les travailleurs.

\subsubsection{Stratégie salariale des firmes en monopsone en horizon infini}

Prenons d'abord, pour bien comprendre le rôle des différents éléments, le cas où l'horizon est infini et les travailleurs n'ont pas la possibilité de changer d'emploi en réponse à une meilleure opportunité salariale (absence de mobilité d'emploi à emploi). Cette économie est l'économie de monopsone décrite par Diamond (1971). Dans cette économie, le salaire n'affecte pas la probabilité d'embauche, puisque tous les travailleurs qui cherchent un emploi sont au chômage, il n'affecte pas non plus la durée de l'emploi, puisque les travailleurs ne démissionnent pas. Le seul effet du salaire est donc de réduire le profit instantané des firmes. Toutes les firmes décideront donc d'offrir le coût d'opportunité du travail (le salaire minimum s'il existe). Le pouvoir de monopsone des firmes est complet et les travailleurs n'ont aucun pouvoir de marché.

Toujours en horizon infini, lorsque les travailleurs peuvent chercher tout en occupant un emploi (présence de mobilité d'emploi à emploi), le pouvoir de monopsone des firmes baisse et les firmes se livrent une véritable concurrence. En effet, la probabilité de recrutement d'un travailleur et la durée de l'emploi seront d'autant plus grandes que la firme offre un salaire haut par rapport aux autres firmes. Cette relation est renforcée par l'ajout de l'hétérogénéité des appariements qui vient accentuer la concurrence pour augmenter la durée de l'emploi. Dans ce jeu désormais, le seul pouvoir qu'ont les firmes et qui permet d'abaisser l'intensité de la concurrence provient des frictions. Les frictions génèrent une masse de chômeurs pour lesquelles il n'est pas utile de se battre (ils acceptent tous les salaires) ainsi qu'un temps de latence avant que les travailleurs trouvent une meilleure opportunité que leur salaire courant.

\subsection{2. Évolution du rapport de force en fonction de l'âge des travailleurs}

5 Afin de garantir l'équiprofit quel que soit le salaire offert 
Au cours du cycle de vie, de par les mobilités vers l'emploi, nous avons vu que le salaire des travailleurs augmentait et que le nombre de travailleurs au chômage diminuait. Si on permet aux firmes de différencier leurs offres selon l'âge alors l'intensité de la concurrence sera plus forte sur le segment des travailleurs seniors, ou en d'autres termes les firmes auront moins de pouvoir de monopsone sur le segment des seniors. La moyenne des salaires offert sera alors plus forte parmi les seniors. Toutefois cet effet est nuancé par le court horizon des travailleurs seniors. En effet, ce court horizon rend inutile la concurrence pour allonger la durée de son emploi puisque quel que soit le niveau de son salaire par rapport aux autres, le travailleur partira prochainement de façon exogène.

\subsubsection{Effet de l'accumulation de capital humain général}

Dans ce modèle, nous prenons en compte l'accumulation de capital humain général en considérant que l'investissement initial du début d'appariement est moindre chez les seniors pour obtenir la même productivité que chez les juniors. En d'autres termes les travailleurs ayant déjà une certaine expérience ont moins besoin d'être formés que les autres pour atteindre la même productivité au sein de l'appariement. Quelle conséquence a cette hypothèse sur le jeu salarial ?

Les firmes ciblant les travailleurs seniors possèdent du fait de cette hypothèse une plus grande marge de manœuvre pour se faire concurrence car elles peuvent créer d'avantage d'emplois très productifs. Cette hypothèse implique donc un renforcement de la concurrence et une hausse des offres de salaires sur le segment des seniors.

\subsection{4. Équilibre}

Dans ce modèle, les valeurs d'équilibre à définir sont des distributions de salaires. On recherche donc un ensemble de fréquences associées à chaque niveau de salaire permettant de retracer des distributions de salaire. L'équilibre consiste à déterminer pour chaque âge, la distribution des salaires offerts par les firmes et celle des travailleurs employés. Ces quatre distributions (deux par âge) influent les unes sur les autres. L'équilibre est atteint lorsque la stationnarité des quatre distributions est obtenue.

\section{Section2 : Un exercice de calibration sur données américaines}

\subsection{Les données}

Nous calibrons le modèle sur données américaines. Ces données sont issues de l'enquête Current Population Survey de 2002. Tous les salaires sont des salaires horaires et sont exprimés en salaire horaire minimum fédéral. Nous ne gardons que les travailleurs salariés ${ }^{6}$ masculins entre 20 et 66 ans. La première période comprend ainsi les travailleurs de 20 à 43 ans et la deuxième période les travailleurs de 43 à 66 ans. Nous prenons en compte les hommes salariés quelque soit leur contrat de travail (temps de travail et durée du contrat). Dans le modèle théorique, les travailleurs entrant sur le marché du travail sont homogènes. Cela suggère que le modèle est plutôt adapté à reproduire la distribution des salaires de travailleurs ayant une même formation initiale. Nous chercherons ainsi à reproduire d'abord la distribution des salaires des travailleurs ayant un même diplôme ${ }^{7}$, ici le high school degree ${ }^{8}$ (cf. figure 2), puis celle de la population totale (cf. figure 1).

Figure 2

Distribution des salaires des travailleurs titulaires d'un high school degree

\footnotetext{
${ }^{6}$ Nous excluons donc toutes les professions indépendantes de type entrepreneurs, artisans, ou professions libérales.

7 Ces travailleurs sont uniquement titulaires de ce diplôme et ne sont titulaires d'aucun autre diplôme supérieur à celui-ci.

${ }^{8}$ Il est possible d'associer le high school degree américain au baccalauréat français.
} 


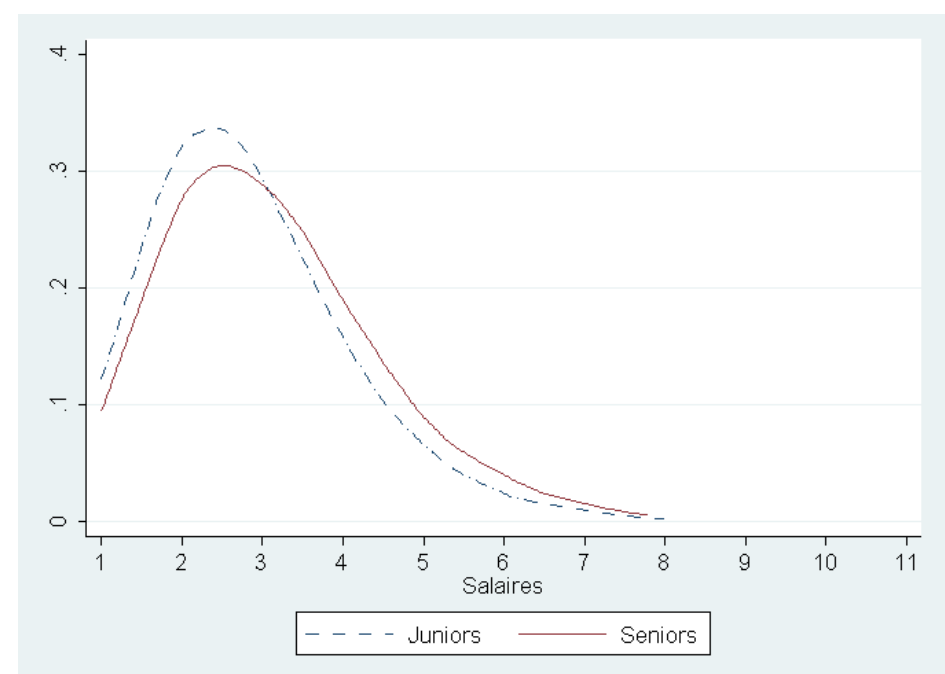

Lecture : les juniors sont constitués des 20-43 ans et les seniors des 43-66 ans . Les salaires sont des salaires horaires et sont exprimés relativement au niveau du salaire horaire minimum fédéral. Champ : hommes salariés, tout contrat, titulaires du high school degree et âgés de 20 à 66 ans. Source : Current Population Survey de 2002.

\subsection{Calibration sur les travailleurs titulaires du high school degree}

Sept paramètres de ce modèle doivent être calibrés.

A La fonction de production est composée de trois paramètres: un paramètre de production fixe, un paramètre pondérant la partie dépendante de l'investissement initial en formation spécifique et enfin l'exposant de cet investissement représentant son rendement.

A L'accumulation de capital humain général est représenté par un paramètre réduisant le coût de l'investissement initial en formation spécifique dans l'appariement.

A Il existe deux probabilités de contact : la probabilité qu'un chômeur entre en contact avec une firme, et celle qu'un employé entre en contact avec une firme. Les probabilités qu'ont les firmes de contacter un travailleur au chômage ou déjà en emploi peuvent être directement déduites de ces deux probabilités.

A Il existe un taux de destruction exogène.

Le premier paramètre de la fonction de production est calibré sur la moyenne des salaires. Les deux autres paramètres, le paramètre de capital humain général et la probabilité de contact entre firmes et employés sont calibrés afin de reproduire la forme de la distribution à travers quatre ratios : le rapport de la médiane sur le premier décile, de la médiane sur le premier quartile, du neuvième décile sur la médiane, et du troisième quartile sur la médiane. Enfin le taux de destruction exogène cherche à reproduire le taux de chômage, et la probabilité de contact entre les firmes et les chômeurs, la durée moyenne de chômage.

Des moments supplémentaires en particulier sur les distributions par âge permettent d'évaluer le modèle. Notons que tous les paramètres du modèle cherchent à reproduire des moments de la distribution agrégée, la différence de distribution entre les deux âges que nous observons est donc entièrement générée par le modèle. Les moments sur les distributions par âge sont le rapport des moyennes, des médianes et des variances entre les seniors et les juniors. Sur la distribution agrégée, nous utilisons les rapports inter quartiles du début et de la fin de la distribution. Enfin j'évalue l'écart entre l'écart-type des salaires mesuré grâce aux données et celui généré par le modèle.

2.2.1. L'échec du modèle de Burdett et Mortensen (1998) en horizon fini à reproduire l'évolution de la distribution de salaires 
Nous testons d'abord le modèle de Burdett et Mortensen (1998) en horizon fini. Nous calibrons pour cela le modèle sans accumulation de capital humain général sur les moments agrégés de la distribution des salaires. Nous testons ensuite la capacité de ce modèle calibré à reproduire des résultats crédibles sur les distributions de salaires par âge : les rapports de moyennes, médianes et variances. Ce modèle ne parvient pas à reproduire le déplacement de la distribution des salaires vers la droite : le modèle explique seulement $5 \%$ de la hausse du salaire moyen avec l'âge et ne parvient pas à reproduire la forme de la distribution observée des salaires. Dans le cadre de ce modèle, à cause de leur horizon court, les firmes sont donc trop réticentes à créer des emplois hautement productifs pour les seniors. ${ }^{9}$

2.2.2. Calibration du modèle avec accumulation de capital humain sur les moments de la distribution des salaires de travailleurs titulaires du high school degree

Nous calibrons donc le modèle complet sur ces mêmes données agrégées. Les cibles de calibration du modèle se trouvent en tableau 1.

Tableau 1

Caractéristiques observées et simulées de la distribution des salaires

Modèle avec accumulation de capital humain

Population des travailleurs titulaires du high school degree

\begin{tabular}{|l|r|r|r|r|r|r|r|r|}
\hline $\begin{array}{l}\text { Moments } \\
\text { cibles }\end{array}$ & Médiane & D5/D1 & D5/Q1 & D9/D5 & Q3/D5 & Moyenne & $\begin{array}{l}\text { Taux de } \\
\text { chômage }\end{array}$ & $\begin{array}{l}\text { Durée de } \\
\text { chômage }\end{array}$ \\
\hline Données & 2,73 & 1,8 & 1,34 & 1,74 & 1,33 & 2,93 & $5 \%$ & 4 mois \\
\hline Modèle & 2,69 & 1,75 & 1,34 & 1,8 & 1,38 & 2,94 & $5,00 \%$ & 4,5 mois \\
\hline
\end{tabular}

Lecture : les salaires sont des salaires horaires et sont exprimés relativement au niveau du salaire horaire minimum fédéral.

Champ : hommes en emploi, tout contrat, titulaires du high school degree et âgés de 20 à 66 ans.

Sources : Current Population Survey de 2002, et calculs à partir du modèle.

Le tableau 2 montre la capacité du modèle à répliquer l'évolution de la distribution des salaires au cours du cycle de vie et à expliquer la dispersion globale des salaires.

Tableau 2

Caractéristiques observées et simulées de la distribution des salaires

Modèle avec accumulation de capital humain

Population des trvailleurs titulaires du high school degree

\begin{tabular}{|l|l|l|l|l|l|l|}
\hline $\begin{array}{l}\text { Moments } \\
\text { résultats }\end{array}$ & $\begin{array}{l}\text { Ratio des } \\
\text { moyennes }\end{array}$ & $\begin{array}{l}\text { Ratio des } \\
\text { médianes }\end{array}$ & $\begin{array}{l}\text { Ratio des } \\
\text { variances }\end{array}$ & Wmax/Q3 & Q1/Wmin & Ecart-type \\
\hline Données & 1,12 & 1,15 & 1,09 & 2,21 & 2,04 & 1,23 \\
\hline Modèle & 1,12 & 1,11 & 1,21 & 1,71 & 2,01 & 1,22 \\
\hline
\end{tabular}

Lecture : les salaires sont des salaires horaires et sont exprimés relativement au niveau du salaire horaire minimum fédéral. Les ratio sont les rapports entre les moments de la distribution des salaires des seniors (43-66 ans) et celles des juniors (20-43 ans).

Champ : hommes en emploi, tout contrat, titulaires (uniquement) du « high school degree » et âgés de 20 à 66 ans.

Sources : Current Population Survey de 2002, et calculs à partir du modèle.

9 Cette spécification du modèle n'étant pas retenue compte tenu du peu de moments qu'elle permet de reproduire (ni progression salariale par âge, ni forme de la distribution), nous choisissons de ne pas en présenter les autres résultats (autres moments et densité générés). 
Le modèle parvient assez bien à reproduire l'évolution des salaires moyens des travailleurs. La variance des salaires des seniors est par contre un peu surestimée par rapport à celle des juniors. L'écart-type des salaires tous âges confondus semble être bien expliqué par le modèle. Toutefois, on observe que le salaire maximal reste sous-estimé, la queue de droite semble tronquée dans le modèle. La distribution générée par le modèle se trouve en figure 3.

Figure 3

Distribution des salaires calculée par le modèle

Modèle avec accumulation de capital humain

Population des travailleurs titulaires du high school degree

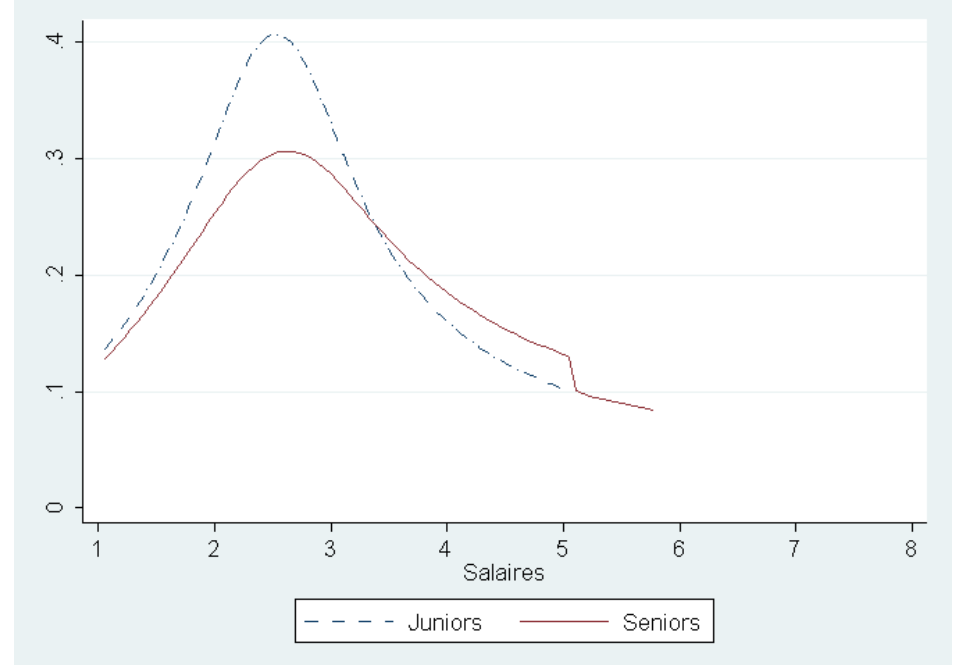

Lecture : les juniors sont constitués des 20-43 ans et les seniors des 43-66 ans . Les salaires sont des salaires horaires et sont exprimés relativement au niveau du salaire horaire minimum fédéral.

Champ : hommes en emploi, tout contrat, titulaires du high school degree et âgés de 20 à 66 ans.

Sources : calculs à partir du modèle.

Il est possible d'évaluer la part de la dispersion expliquée par l'accumulation de capital humain général dans le cas particulier de cette calibration. Pour cela, nous laissons tous les autres paramètres inchangés et retirons le paramètre associé à l'accumulation de capital humain (cf. Figure 4).

Figure 4

Distribution des salaires calculée par le modèle

Modèle sans accumulation de capital humain

Population des travailleurs titulaires du high school degree 


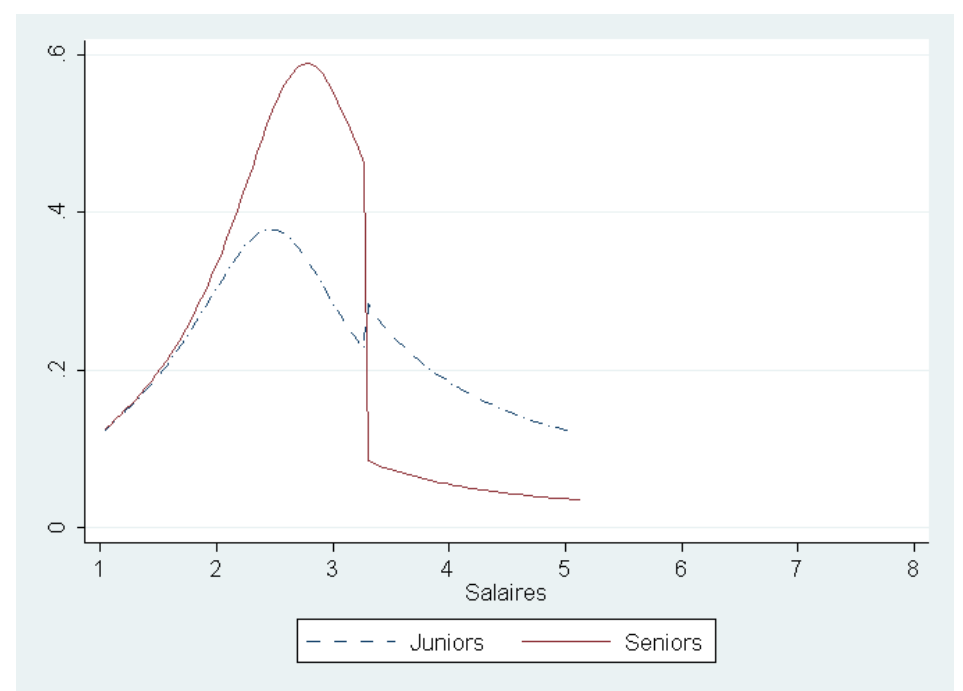

Lecture : les juniors sont constitués des 20-43 ans et les seniors des 43-66 ans . Les salaires sont des salaires horaires et sont exprimés relativement au niveau du salaire horaire minimum fédéral. Champ : hommes en emploi, tout contrat, titulaires du high school degree et âgés de 20 à 66 ans. Sources : calculs à partir du modèle.

Cette simulation montre l'importance du capital humain dans nos résultats. De plus, nous nous sommes assurés que pour l'ensemble des valeurs admissibles des paramètres, il n'était pas possible avec le modèle sans capital humain de reproduire autant de moments qu'avec le modèle complet ${ }^{10}$. Sur la figure 4, nous observons que les firmes réservent les salaires supérieurs à 3,5 aux travailleurs juniors qui sont plus prometteurs en termes d'horizon. Les travailleurs seniors semblent eux recevoir plus de salaires compris entre 2 et 3,5, ce qui s'explique par leur plus grand pouvoir de marché sur les firmes. Sur la figure 3, les travailleurs seniors perçoivent relativement plus de salaires au dessus de 3,5 et les inexpérimentés plus de salaires inférieurs à cette limite. Cette comparaison nous enseigne donc que si les relativement hauts salaires des seniors proviennent essentiellement d'un rapport de force avec les firmes plus en leur faveur, le fait qu'ils détiennent la majorité des plus hauts salaires est le résultat de leur meilleure productivité (plus de capital humain général). Sans accumulation de capital humain général, les seniors seraient certes, relativement aux juniors, à l'abri des plus bas salaires, mais n'auraient que peu accès aux très hauts salaires.

2.3. Calibration du modèle avec accumulation de capital humain sur les moments de la distribution des salaires de la population totale

Il est également possible d'effectuer une calibration sur la population totale, c'est-à-dire toutes formations initiales confondues. Les résultats de la calibration se trouvent dans le tableau 3 et la distribution générée par le modèle en figure 5.

Tableau 3

Caractéristiques observées et simulées de la distribution des salaires

Modèle avec accumulation de capital humain

Population des travailleurs toutes formations initiales confondues

\begin{tabular}{|l|r|r|r|r|r|r|r|r|}
\hline $\begin{array}{l}\text { Moments } \\
\text { cibles }\end{array}$ & Médiane & D5/D1 & D5/Q1 & D9/D5 & Q3/D5 & Moyenne & $\begin{array}{l}\text { Taux de } \\
\text { chômage }\end{array}$ & $\begin{array}{l}\text { Durée de } \\
\text { chômage }\end{array}$ \\
\hline Données & 3,16 & 1,89 & 1,41 & 1,87 & 1,38 & 3,52 & $5 \%$ & 4 mois \\
\hline
\end{tabular}

${ }^{10}$ Voir sous-section intitulée : L'échec du modèle de Burdett et Mortensen (1998) en horizon fini à reproduire l'évolution de la distribution de salaires 


\begin{tabular}{|c|c|c|c|c|c|c|c|c|}
\hline Modèle & 3,2 & 1,88 & 1,38 & 1,74 & 1,35 & 3.5 & $4.80 \%$ & 4 mois \\
\hline
\end{tabular}

Lecture : les salaires sont des salaires horaires et sont exprimés relativement au niveau du salaire horaire minimum fédéral.

Champ : hommes en emploi, tout contrat, âgés de 20 à 66 ans.

Sources : Current Population Survey de 2002, et calculs à partir du modèle.

Figure 5

Distribution des salaires calculée par le modèle

Modèle avec accumulation de capital humain

Population des travailleurs toutes formations initiales confondues

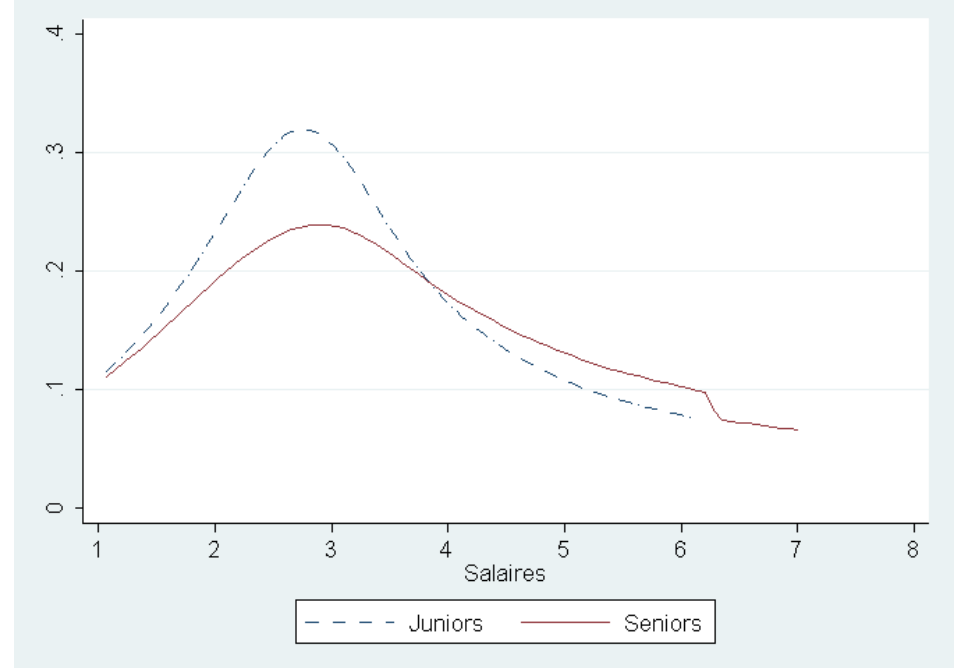

Lecture : les juniors sont constitués des 20-43 ans et les seniors des 43-66 ans . Les salaires sont des salaires horaires et sont exprimés relativement au niveau du salaire horaire minimum fédéral.

Champ : hommes en emploi, tout contrat, âgés de 20 à 66 ans.

Sources : calculs à partir du modèle.

Le tableau 4 présente la capacité du modèle à répliquer les moments supplémentaires, quand on étudie la population totale.

Tableau 4

Caractéristiques observées et simulées de la distribution des salaires

Modèle avec accumulation de capital humain

Population des travailleurs toutes formations initiales confondues

\begin{tabular}{|l|l|l|l|l|l|l|}
\hline $\begin{array}{l}\text { Moments } \\
\text { résultats }\end{array}$ & $\begin{array}{l}\text { Ratio des } \\
\text { moyennes }\end{array}$ & $\begin{array}{l}\text { Ratio des } \\
\text { médianes }\end{array}$ & $\begin{array}{l}\text { Ratio des } \\
\text { variances }\end{array}$ & Wmax/Q3 & Q1/Wmin & Ecart-type \\
\hline Données & 1,12 & 1,13 & 1,11 & 2,53 & 2,24 & 1,71 \\
\hline Modèle & 1,13 & 1,14 & 1,21 & 1,62 & 2,27 & 1,42 \\
\hline
\end{tabular}

Lecture : les juniors sont constitués des 20-43 ans et les seniors des 43-66 ans . Les salaires sont des salaires horaires et sont exprimés relativement au niveau du salaire horaire minimum fédéral.

Champ : hommes en emploi, tout contrat, âgés de 20 à 66 ans.

Sources : Current Population Survey de 2002 et calculs à partir du modèle.

La population totale est plus hétérogène. Le mode est légèrement plus écrasé et la queue de distribution est plus allongée. Cette calibration permet de rendre compte de la forme un peu plus écrasée du mode, marque d'une plus grande dispersion autour du mode induite par la plus grande 
hétérogénéité des travailleurs. Toutefois, la population totale a aussi la caractéristique de présenter une plus grande dispersion en queue de distribution pour la même raison. Cette dispersion semble plus difficilement reproductible par le modèle. Plus la population est hétérogène, plus il est difficile avec ce modèle de rendre compte de la dispersion totale des salaires.

\section{Section 3 : Conclusion et perspectives}

Le modèle de Burdett et Mortensen (1998) en horizon fini ne permet pas d'expliquer l'évolution de la distribution des salaires au cours du cycle de vie. Le mécanisme manquant est celui de l'accumulation de capital humain général. Dans cet article, en introduisant une accumulation exogène de capital humain général, nous montrons que ce mécanisme contribue à la dispersion totale des salaires et permet de reproduire correctement l'évolution du jeu de salaires avec l'âge.

Un aspect lié à l'âge n'a cependant pas été pris en compte dans cet article si on considère que les firmes peuvent entrer librement sur les deux marchés : Le profit engendré par le recrutement d'un senior sera très probablement différent de celui engendré par le recrutement d'un junior et donc le nombre d'emplois vacants pourra être différent. A l'équilibre, une condition de libre entrée pourra affecter la tension sur le marché du travail, et donc les probabilités de mobilité et le rapport de force entre les firmes et les travailleurs.

Les extensions possibles de ce cadre théorique sont multiples, notamment pour l'évaluation de politiques économiques. Quantité d'institutions influençant le marché du travail ont des effets différenciés en fonction des âges des travailleurs. Ce modèle peut contribuer à en évaluer les effets sur l'emploi, la distribution des salaires et la mobilité des travailleurs.

L'auteur remercie François Langot et Jean-Olivier Hairault pour leurs remarques et suggestions. Ce travail a aussi bénéficié des commentaires de deux rapporteurs anonymes de la Revue Française d'Économie.

Sarah LE DUIGOU est doctorante en économie à l'Université du Maine au sein du laboratoire GAINS-TEPP (Université du Maine et CNRS) sous la direction du professeur François Langot. Adresse : Université du Maine, avenue O. Messiaen, 72085 Le Mans cedex

Courriel : sarah.leduigou@gmail.com

\section{REFERENCES:}

D. Acemoglu et R. Shimer [1999]: Holdups and Efficiency with Search Frictions, Labour Economics, 40, pp. 827-849.

J. Bagger, F.Fontaine, F. Postel-Vinay et J.-M. Robin [2009]: A Feasible Equilibrium Search Model of Individual Wage Dynamics with Experience Accumulation, Centre for structural Econometrics, University of Bristol, Discussion paper 09/02.

K. Burdett et M. Coles [2003]: Equilibrium wage-tenure contracts, Econometrica, Econometric society, 71(5), pp. 1377-1404.

K. Burdett et D.T. Mortensen [1998]: Wage Differentials, Employer Size, and Unemployment, International Economic Review,39, pp. 257-273.

K. Burdett et K. Judd [1983], Equilibrium Price Dispersion, Econometrica, 51, pp.955-969.

GR. Butters [1977]: Equilibrium Distributions of Sales and Adverstising Prices, The Review of Economic Studies, 44, pp. 465-491. 
P.A. Diamond [1971]: A model of price adjustment, Journal of Economic Theory, 3(2), pp 156168.

G. Menzio, I. A. Telyukova et L.Visschers [2012]: Directed Search over the Life Cycle, PIER Working Paper Archive 12-002, Penn Institute for Economic Research, University of Pennsylvania.

D.T. Mortensen [1990]: On-the-Job Search and the Wage Distribution, Journal of Labor Economics, 23, pp. 32-58.

D.T. Mortensen [1998] : Equilibrium Unemployment with Wage Posting: Burdett-Mortensen Meet Pissarides, 98-14, Centre for Labour Market and Social Research, Danmark.

F. Postel-Vinay et J.-M. Robin [2002]: The Distribution of Earnings in an Equilibrium Search Model with State-Dependent Offers and Counter-Offers, International Economic Review, 43(4), 989-1016. 